Bull. Austral. Math. Soc.

VoL. 55 (1997) [1-5]

\title{
NOTE ON A THEOREM OF GROMOLL-GROVE
}

\author{
Grant Cairns and Richard H. Escobales, Jr.
}

\begin{abstract}
D. Gromoll and K. Grove showed that metric flows on constant curvature spaces are either flat or locally spanned by Killing vector fields. We generalise this result to certain flows on manifolds of variable curvature.
\end{abstract}

Gromoll and Grove's 1985 theorem [5] asserts that on manifolds of constant curvature, a metric flow is either flat or locally homogeneous. Recently this was used as the key ingredient in the classification of metric flows on spaces of constant nonpositive curvature [1]. Basmajian and Walschap's paper concludes with the remark: "it is interesting to note that many of the above arguments carry over to manifolds with curvature bounded above by a negative constant... The proof of the Gromoll-Grove theorem, however, makes extensive use of constant curvature". In this note we generalise the Gromoll-Grove theorem to spaces of variable curvature.

Let $(M, g)$ be a complete, connected $C^{\infty}$ Riemannian manifold with metric flow $\mathcal{F}$ generated by a unit vector field $V$, which spans the vertical distribution $\mathcal{V}$. The "metric" hypothesis means that the flow lines are locally equi-distant [10]. The flow is flat if the horizontal distribution $\mathcal{H}=\mathcal{V}^{\perp}$ is integrable. The flow is locally homogeneous if it is locally spanned by Killing vector fields. Notice that the two conditions are of quite different natures: flatness only depends on the decomposition $T M=\mathcal{H} \oplus \mathcal{V}$ of the tangent bundle and it is a strong constraint on the topology of $M$, whereas local homogeneity depends on the parametrisation of $\mathcal{F}$ and on compact manifolds every metric flow is locally homogeneous for some choice of metric [3]. The two conditions nevertheless have one point in common: it is possible to express them as invariance conditions "along the flow lines" of $\mathcal{F}$. So the obvious task is to replace the global constant curvature hypothesis by an invariance condition, along the flow lines. Recall (see [7]) that a differential form $\alpha$ on $M$ is basic if it is locally projectable along the flow lines (this means that $0=\alpha(V)=d \alpha(V,$.$) ). A vector field X$ is foliate if it is locally projectable along the flow lines (this means that $[V, X]$ is vertical). We say that $X$ is basic if it is foliate and horizontal [4].

Received 30th January, 1996.

This paper was completed while the second author was at La Trobe University on sabbatical leave from Canisius College. He wishes to thank La Trobe for its hospitality in making his stay there both pleasant and possible and both institutions for their support.

Copyright Clearance Centre, Inc. Serial-fee code: 0004-9729/97 \$A2.00+0.00. 
THeOREM. Suppose that the curvature tensor $R$ satisfies the following condition: (*) $R_{X Y} Z$ is basic, for all basic local vector fields $X, Y, Z$. Then $\mathcal{F}$ is either locally homogeneous or flat.

Proof: The Gromoll-Grove proof in [5] can be broken down into 3 parts:

LEMMA 1. If $\mathcal{H}$ is integrable at one point, then it is integrable everywhere.

LEMMA 2. If $\mathcal{H}$ is not integrable, then $\mathcal{F}$ is tense (that is, the mean curvature one-form $\kappa$ is basic).

LEMMA 3. If $\mathcal{F}$ is tense, then $\kappa$ is closed.

For any vector field $E, \kappa(E)=g\left(E, T_{V} V\right)$, where $T$ is the second fundamental form tensor, as in [9] or [4]. Equivalently $\kappa$ can be defined by the equations $\kappa(V)=0$ and $\kappa(X)=g([X, V], V)$ when $X$ is horizontal.

We shall show that the above lemmas follow from (*). The theorem now follows, since by following [5] it is easy to see that for arbitrary metric flows, $\kappa$ closed is equivalent to $\mathcal{F}$ locally homogeneous. First notice that $(*)$ can be written as two conditions:

$$
\begin{aligned}
& g\left(R_{X Y} Z, W\right) \text { is basic, for all basic local vector fields } X, Y, Z, W \\
& g\left(R_{X Y} Z, V\right)=0, \text { for all basic local vector fields } X, Y, Z
\end{aligned}
$$

For convenience, we introduce the 2 -form $\Omega$ which is zero on $V$ and for which $\Omega(X, Y)=$ $g([X, Y], V)$, for all horizontal $X$ and $Y$. (Notice that $\Omega(X, Y) . V=A_{X} Y / 2$, where $A$ is the integrability tensor. See [9] or [8].) Our proof uses the work of O'Neill [9]. From [9, Theorem 2, Equation $\{4\}$ ] and [9, Corollary 1, Equation 3], it follows that (1) is equivalent to the following condition:

$$
\Omega \text { is basic; that is } V \Omega(X, Y)=0 \text {, for all basic local vector fields } X, Y \text {. }
$$

On the other hand, rewriting [9, Equation $\{3\}],(2)$ is equivalent to:

$$
\begin{aligned}
0= & Z \Omega(X, Y)-\Omega\left(\nabla_{Z} X, Y\right)-\Omega\left(X, \nabla_{Z} Y\right) \\
& -\Omega(X, Y) \kappa(Z)+\Omega(Y, Z) \kappa(X)+\Omega(Z, X) \kappa(Y)
\end{aligned}
$$

To prove Lemma 1 , notice that if $\Omega$ vanishes at some point $q$, then by (3), $\Omega$ vanishes on the flow line of $\mathcal{F}$ through $q$. Transverse to $\mathcal{F}$, equation (4) establishes a system of linear equations for $\Omega$ having zero initial condition at each point of the flow line. A connectedness argument concludes the proof.

We now prove Lemma 2. Assume that $\mathcal{H}$ is not integrable. By Lemma $1, \Omega$ is nowhere zero. Let $q \in M$ and choose basic local vector fields $Y$ and $Z$ so that 
$\Omega(Y, Z) \neq 0$ at $q$. Now let $X$ be another basic local vector field on some neighbourhood of $q$. By (3), all but the last three terms of (4) are constant on the plaques of $\mathcal{F}$. So applying $V$ to (4) gives

$$
0=-\Omega(X, Y) V \kappa(Z)+\Omega(Y, Z) V \kappa(X)+\Omega(Z, X) V \kappa(Y) .
$$

Similarly, by cyclically permuting $X, Y, Z$ one gets

$$
0=-\Omega(Z, X) V \kappa(Y)+\Omega(X, Y) V \kappa(Z)+\Omega(Y, Z) V \kappa(X)
$$

Adding (5) and (6) gives $\Omega(Y, Z) V \kappa(X)=0$. So, as $\Omega(Y, Z) \neq 0$, one has $V \kappa(X)=0$; that is, $\kappa(X)$ is basic. Since $X$ was arbitrary, we conclude that $\kappa$ is basic.

Finally, we prove Lemma 3 . This result is true on compact manifolds, by a result of Kamber and Tondeur (see [11]). More generally, the result follows easily from (*), as we now show. Suppose that $\kappa$ is basic and let $X, Y$ be local basic vector fields. Clearly $d \kappa(X, V)=0$. To show that $\kappa$ is closed, we need to establish that $d \kappa(X, Y)=0$. Now from [4] (a shorter proof is given in [2]), $d \kappa(X, Y)=-\operatorname{div}_{\mathcal{F}} \mathcal{V}[X, Y]$, where $\mathcal{V}[X, Y]$ is the vertical component of $[X, Y]$ and $\operatorname{div}_{\mathcal{F}}$ is the divergence in the flow lines of $\mathcal{F}$. Writing $\mathcal{V}[X, Y]=\Omega(X, Y) . V$ gives

$$
d \kappa(X, Y)=-\operatorname{div}_{\mathcal{F}} \mathcal{V}[X, Y]=-g([\mathcal{V}[X, Y], V], V)=V \Omega(X, Y) .
$$

Hence the required result follows immediately from (3). This completes the proof of the theorem.

REMARK. Conversely, if $\mathcal{F}$ is a flat metric flow, then $\Omega \equiv 0$ and so by (3) and (4), condition $(*)$ is satisfied. On the other hand, if $\mathcal{F}$ is locally homogeneous, then it doesn't necessarily follow that $(*)$ holds, as we see in Example 3 below.

\section{EXAMPLES}

The following examples are "algebraic". Let $\mathcal{G}$ be a simply connected Lie group with Lie algebra $g$ of left invariant vector fields, and suppose that $\mathcal{G}$ possesses a discrete subgroup $\Gamma$, with quotient $M=\Gamma \backslash G$. Then $\mathfrak{g}$ induces a Lie algebra of nonsingular vector fields on $M$. The flows defined by these vector fields are said to be algebraic. Now choose a scalar product on $\mathfrak{g}$ and equip $M$ with the induced Riemannian metric $g$. Very few of the resulting manifolds have constant curvature (see [6]). In the following examples, we use the same symbols to denote the elements of $g$ and the corresponding vector fields on $M$. 
EXAMPLE 1. Let $\mathfrak{g}$ be the Heisenberg Lie algebra with orthonormal basis $X, Y, V$ and relation $[X, Y]=V$. It is easy to see that $V$ defines a metric flow and $X$ and $Y$ are basic vector fields. Using the Levi-Civita formula for the covariant derivative of left-invariant vector fields,

$$
2 g\left(\nabla_{E} F, G\right)=g([E, F], G)-g(F,[E, G])-g(E,[F, G]),
$$

one sees that

$$
\begin{array}{ll}
\nabla_{X} X=\nabla_{Y} Y=\nabla_{V} V=0, & \nabla_{X} Y=-\nabla_{Y} X=\frac{1}{2} V, \\
\nabla_{X} V=\nabla_{V} X=-\frac{1}{2} Y, & \nabla_{Y} V=\nabla_{V} Y=\frac{1}{2} X .
\end{array}
$$

Then $R_{X Y} X=-(3 / 4) Y, R_{X, Y} Y=(3 / 4) X$, so (*) holds. This example is clearly not flat, but it is homogeneous $(\kappa \equiv 0)$. (Here we are using the curvature convention of [9], $R_{X Y}=\nabla_{[X, Y]}-\left[\nabla_{X}, \nabla_{Y}\right]$, which is the opposite of that of [5].)

EXAMPLE 2. Let $\mathfrak{g}$ be the algebra $s l(2, \mathbb{R})$ with orthonormal basis $X, Y, V$ and relations $[V, X]=V+Y,[V, Y]=V-X$ and $[X, Y]=-X+Y$. Again $V$ defines a metric flow and $X$ and $Y$ are basic vector fields. Using (7) one has

$$
\begin{array}{lll}
\nabla_{X} V=\nabla_{Y} V=0, & \nabla_{X} X=-\nabla_{Y} X=Y, & \nabla_{Y} Y=-\nabla_{X} Y=X \\
\nabla_{V} X=V+Y, & \nabla_{V} Y=V-X, & \nabla_{V} V=-X-Y .
\end{array}
$$

Then $R_{X Y} X=-2 Y, R_{X, Y} Y=2 X$, so (*) holds. This example is flat but not locally homogeneous (for example $d \kappa(X, V) \neq 0$ ).

EXAMPLE 3. Let $\mathfrak{g}$ be the 4-dimensional nilpotent Lie algebra with orthonormal basis $X, Y, Z, V$ and relations $[X, Y]=Z$ and $[X, Z]=V$. Again $V$ is a metric flow and $X, Y, Z$ are basic vector fields. One has

$$
\begin{aligned}
& \nabla_{X} X=\nabla_{Y} Y=\nabla_{Z} Z=\nabla_{V} V=\nabla_{Y} V=\nabla_{V} Y=0, \\
& \nabla_{X} Y=-\nabla_{Y} X=-\nabla_{X} V=-\nabla_{V} X=\frac{1}{2} Z, \quad \nabla_{X} Z=\frac{1}{2} V-\frac{1}{2} Y, \\
& \nabla_{Z} X=-\frac{1}{2} V-\frac{1}{2} Y, \quad \nabla_{Y} Z=\nabla_{Z} Y=\nabla_{Z} V=\nabla_{V} Z=\frac{1}{2} X .
\end{aligned}
$$

This example is homogeneous, but $R_{X Y} X=-V / 4-(3 / 4) Y$, so (*) fails to hold.

\section{REFERENCES}

[1] A. Basmajian and G. Walschap, 'Metric flows in space forms of nonpositive curvature', Proc. Amer. Math. Soc. 123 (1995), 3177-3181. 
[2] G. Cairns and R. Escobales, Jr, 'Further geometry of the mean curvature one-form and the normal-plane field one-form on a foliated Reimannian manifold', J. Aust. Math. Soc. (to appear).

[3] D. Domínguez, 'Finiteness and tenseness theorems for Riemannian foliations', (preprint).

[4] R. Escobales, Jr. and P. Parker, 'Geometric consequences of the normal curvature cohomology class in umbilic foliations', Indiana Univ. Math. J. 37 (1988), 389-408.

[5] D. Gromoll and K. Grove, 'One-dimensional metric foliations in constant curvature spaces', in Differential geometry and complex analysis (Springer-Verlag, Berlin, Heidelberg, New York, 1985), pp. 165-168.

[6] J. Milnor, 'Curvatures of left invariant metrics on Lie groups', Adv. in Math. 21 (1976), 293-329.

[7] P. Molino, Riemannian foliations (Birkhauser, Boston, Basel, 1988).

[8] T. Nagano, 'On fibred Riemann manifolds', Sci. Papers Gen. Ed. Univ. Tokyo 10 (1960), 17-27.

[9] B. O'Neill, 'The fundamental equations of a submersion', Michigan Math. J. 13 (1966), 459-469.

[10] B. Reinhart, 'Foliated manifolds with bundle-like metrics', Ann. of Math. 69 (1959), 119-131.

[11] P. Tondeur, Foliations on Riemannian Manifolds (Springer-Verlag, Berlin, Heidelberg, New York, 1988).

School of Mathematics

La Trobe University

Melbourne Vic. 3083

Australia

e-mail: G.Cairns@latrobe.edu.au

\author{
Department of Mathematics \\ Canisius College \\ Buffalo, NY 14208 \\ United States of America \\ e-mail: escobalr@gort.canisius.edu
}

